\title{
Collinear terahertz generation in photonic crystal structures via difference-frequency generation
}

\author{
Nadia Mattiucci \\ Time Domain Corporation, Cummings Research Park, 7097 Old Madison Pike, Huntsville, Alabama 35806, Charles \\ M. Bowden Research Center, Research Development and Engineering Command (RDECOM) Building 7804, \\ Redstone Arsenal, Alabama 35898-5000, and Dipartimento di Fisica E. Amaldi, Università RomaTre, \\ Via Della Vasca Navale, 84, I-00146 Rome Italy
}

Giuseppe D'Aguanno

Time Domain Corporation, Cummings Research Park, 7097 Old Madison Pike, Huntsville, Alabama 35806, and Charles M. Bowden Research Center, RDECOM Building 7804, Redstone Arsenal, Alabama 35898-5000

Michael Scalora and Mark J. Bloemer

Charles M. Bowden Research Center, RDECOM Building 7804, Redstone Arsenal, Alabama 35898-5000

Neset Akozbek

Time Domain Corporation, Cummings Research Park, 7097 Old Madison Pike, Huntsville, Alabama 35806

\author{
J. W. Haus \\ Electro-Optics Program, University of Dayton, Dayton, Ohio, 45469-0245
}

Received April 5, 2005; revised July 6, 2005; accepted July 8, 2005

\begin{abstract}
We have developed a three-dimensional model to study the properties of terahertz emission from a onedimensional, $\chi^{(2)}$-doped photonic crystal. We exploit difference-frequency generation in a collinear configuration and find an enhancement factor of up to 20 with respect to difference-frequency conversion from an equivalent bulk structure. (C) 2006 Optical Society of America
\end{abstract}

OCIS codes: 190.0190, 190.2620, 230.4170.

\section{INTRODUCTION}

Recently, a great deal of attention has been devoted to the problem of the generation of coherent terahertz $(\mathrm{THz})$ radiation because of the many possible applications that have been identified: from spectroscopic imaging to radar systems and from security and medical to biological purposes as well as pure research purposes. Unfortunately, the number and types of $\mathrm{THz}$ sources available are not always adequate for all possible applications mentioned. This has led to a great deal of activity to come up with new designs and sources with different characteristics. Free-electron lasers and synchrotron radiation are powerful $\mathrm{THz}$ sources, but their physical size limits their application. More compact sources are based on photoconductivity (electro-optic devices) and optical rectification (all optical devices). In the first case a femtosecond laser operating in the visible or near-infrared regime is used to create electron-hole pairs that accelerate through an appropriate electric field. The resulting changing dipole leads the generation of $\mathrm{THz}$ radiation. ${ }^{1,2}$ These kinds of devices are usually referred to as photoconductive antennas. In the second case a femtosecond pulse interacts with a nonlinear crystal with a second-order nonlinear suscep- tibility, generating a $\mathrm{THz}$ pulse through optical rectification. ${ }^{3}$ Organic and inorganic electro-optic crystals such as $4^{\prime}$-N,N-dimethylamino-4-N-methylstilbazolium toluene- $p$-sulfonate (DAST), ZnTe, GaAs, and $\mathrm{LiNbO}_{3}{ }^{4,5}$ as well as polymers, ${ }^{6}$ have also been exploited. A wide range of techniques are currently under investigation including quantum cascading, ${ }^{7}$ optical parametric oscillations, ${ }^{8}$ and femtosecond pulse shaping. ${ }^{9}$ Most of these sources produce $\mathrm{THz}$ pulses. Continuous $\mathrm{THz}$ sources can be obtained through difference-frequency generation in second-order nonlinear crystals. In this case two cw optical pumps interact in the crystal and generate coherent $\mathrm{THz}$ radiation at frequency $\Delta \omega=\omega_{1}-\omega_{2}$, where $\omega_{1}$ and $\omega_{2}$ are the frequencies of the pumps. For this purpose, inorganic crystals such as $\mathrm{GaP}^{10}$ and $\mathrm{GaSe},{ }^{11}$ for example, have been used. Most recently, organic crystals (DAST) with large nonlinearity have been explored with promising results. ${ }^{12,13}$ Some limitations of those techniques include low tunability range or low efficiency or both. Low efficiency is usually due to material absorption at $\mathrm{THz}$ wavelengths. Typical conversion efficiencies experimentally achieved are of the order $\eta \sim 10^{-6} .11$

In this paper, we study $\mathrm{THz}$ emission from a $\chi^{(2)}$-doped, 
one-dimensional photonic crystal (1-D PC) on the basis of a rigorous Green function approach and a threedimensional (3-D) vector model. $\mathrm{THz}$ radiation is generated via a difference-frequency generation process, in which two nearly degenerate optical pumps of frequencies $\omega_{1}$ and $\omega_{2}$ generate a frequency $\omega_{3}=\omega_{1}-\omega_{2}$ found in the $\mathrm{THz}$ range. The pumps are assumed to work in a collinear configuration. The 1-D PC structures that we study are known to exhibit some peculiar properties, such as field localization, superluminal pulse propagation in the bandgap, and high density of modes, to name a few, but the structure is flexible enough that it can be optimized for various and quite different purposes, including nonlinear frequency conversion. ${ }^{14}$ Previously, the dispersive properties of photonic bandgap structures had been used to solve the phase-matching problem using the optical rectification effect. ${ }^{15}$ The use of 1-D layered structures, in order to generate submillimeter wavelengths through differencefrequency generation, was already anticipated by Bloembergen and Sievers ${ }^{16}$ in 1970 . In their seminal paper, the authors discussed the properties of nonlinear optics, in particular, of second-order phenomena, namely, secondharmonic and sum- and difference-frequency generation in such structures. Although Bloembergen and Sievers considered infinite structures, the results they obtained have general validity: They showed that the periodicity of the layered structure can be used to compensate the normal dispersion of materials in order to obtain high conversion efficiency through phase-matched second-order interactions. On the other hand, as we will see later, in the case of finite structures the efficiency of second-order interaction is not directly linked to a phase-matching term but rather to overlap integrals of the interacting fields, as also discussed in Refs. 17 and 18. A first study of THz emission in 1-D PCs that used a coupled-mode analysis to describe multiple-field confinement, enhancement, and overlap near the photonic band edge of $1-\mathrm{D}$ photonic bandgap structures was described in Ref. 19 That study, which analyzed $\mathrm{THz}$ emission from a defect layer, showed that it was possible, at least in principle, to obtain continuous and tunable sources from the sub-THz regime up to $12 \mathrm{THz}$. The advantages of using finite 1-D PCs to generate $\mathrm{THz}$ radiation can be found in the flexibility of those structures that allow achieving high conversion efficiency either in a collinear or a noncollinear configuration owing to high-field localizations.

The paper is organized as follows. In Section 1 we discuss the properties that a 1-D PC structure should have to efficiently generate $\mathrm{THz}$ radiation. In Sections 2 and 3 we develop a 1-D model and 3-D model, respectively, both based on the Green function theory. The results of the two models are then compared. In Section 4 we study the behavior of $\mathrm{THz}$ generation in a counterpropagating pump configuration.

\section{GENERAL CONSIDERATIONS}

We are studying $\mathrm{THz}$ generation via a second-order process, which means we have two pumps and four secondorder processes involved and four generated fields. Three of those fields are generated at optical frequencies $\left(2 \omega_{1}, 2 \omega_{2}, \omega_{1}+\omega_{2}\right)$, while the fourth is generated in the

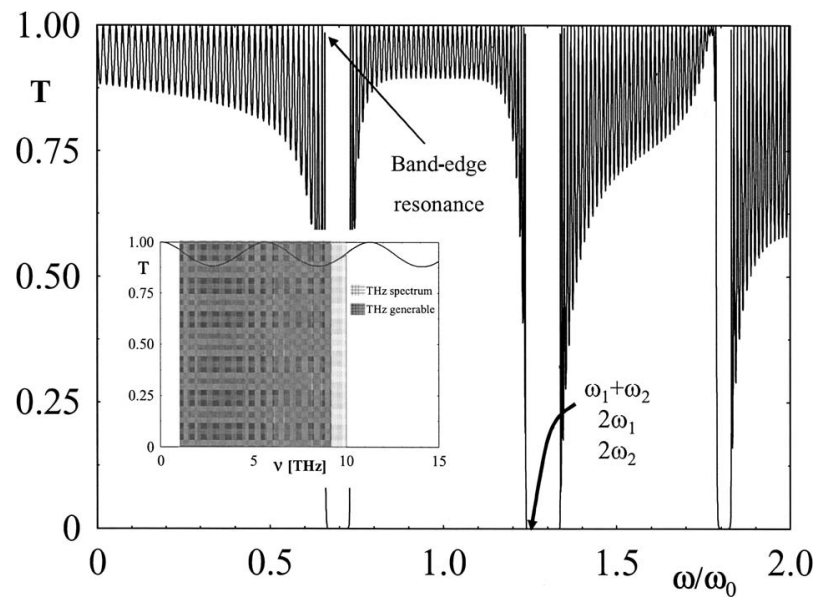

Fig. 1. Transmission spectrum of a 1-D periodic structure. The elementary cell is made by two layers of refractive index $n_{1}$ $=1.8$ and $n_{2}=1.4$ at the frequency $\lambda_{0}=1 \mu \mathrm{m}$. The higher-index layer exhibits a normal dispersion. The thicknesses of the two layers are, respectively, $d_{1}=\lambda_{0} / 2 n_{1}, d_{2}=\lambda_{0} / 4 n_{2}$. The structure is surrounded by air $\left(n_{0}=1\right)$. The transmission is a function of the normalized frequency $\omega / \omega_{0}$, where $\omega_{0}=2 \pi c / \lambda_{0}$. In the inset is reported the spectrum of THz frequencies as a function of THz. The dark region in the inset is the part of the $\mathrm{THz}$ spectrum for which all the undesired second-order processes fall in the gap.

$\mathrm{THz}$ region $\left(\omega_{1}-\omega_{2}\right)$. The efficiency of optical processes is, in general, much greater than $\mathrm{THz}$ generation, and so those processes cannot be ignored. The first challenge is to inhibit any undesired process. The transmission spectrum of a 1-D PC is characterized by wide bandgaps and relatively narrow transmission peaks. Frequencies that fall inside the gap correspond to modes that are not supported by the cavity and have poor localization properties, ${ }^{20}$ so that if a generated frequency falls inside the gap, the related process is practically inhibited. The key to efficient $\mathrm{THz}$ generation is then to find a structure in which all the generated frequencies, except the $\mathrm{THz}$, are tuned inside a transmission gap. The two optical pumps $\left(\omega_{1}\right.$ and $\left.\omega_{2}\right)$ are nearly degenerate $\left(\omega_{1} \sim \omega_{2} \sim \omega\right)$; therefore, one important condition is to design a 1-D structure such that the frequency $2 \omega$ happens to fall inside a gap.

As an example, in Fig. 1 we show the transmittance of a 1-D structure composed of 40 periods of alternating layers $\left(n_{1} \sim 1.8, n_{2} \sim 1.4\right)$ whose thicknesses are chosen to be $a=\lambda / 2 n_{1}$ and $b=\lambda / 4 n_{2}$, respectively, where $\lambda$ is the reference wavelength $\lambda=1 \mu \mathrm{m}$. The high-index layers are assumed to possess normal dispersion, whereas, for simplicity, the low-index material is assumed to be dispersionless. Figure 1 thus suggests that if the pumps $\omega_{1}$ and $\omega_{2}$ are chosen near the first-order band edge, as indicated, then the frequencies $2 \omega_{1}, 2 \omega_{2}$, and $\omega_{1}+\omega_{2}$ are to be found somewhere inside the second-order bandgap, and their generation will be suppressed. On the other hand, the wave at the difference frequency $\omega_{1}-\omega_{2}(\mathrm{THz})$ will be tuned within the first passband, away from any gap, and so it will not be inhibited.

\section{PLANE-WAVE APPROACH}

In this section we follow a plane-wave approach to describe the multiwave interaction. We deal with a six-field 
problem: two pumps ( $\omega_{1}$ and $\omega_{2}$ ), the second harmonics ( $2 \omega_{1}$ and $\left.2 \omega_{2}\right)$, the upconverted or sum frequency $\left(\omega_{1}\right.$ $\left.+\omega_{2}\right)$, and the downconverted or difference frequency $\left(\omega_{1}\right.$ $\left.-\omega_{2}\right)$. We have to solve a system of six coupled, secondorder differential equations:

$$
\begin{aligned}
& \frac{\mathrm{d}^{2}}{\mathrm{~d} z^{2}} E_{\omega_{1}}+\frac{\omega_{1}^{2} \epsilon_{\omega_{1}} E_{\omega_{1}}}{c^{2}}=-2 \frac{\omega_{1}^{2}}{c^{2}}\left(d^{(2)} E_{\omega_{1}}^{*} E_{2 \omega_{1}}+d^{(2)} E_{\omega_{2}}^{*} E_{\omega_{1}+\omega_{2}}\right. \\
& \left.+d^{(2)} E_{\omega_{2}} E_{\omega_{1}-\omega_{2}}\right) \\
& \frac{\mathrm{d}^{2}}{\mathrm{~d} z^{2}} E_{\omega_{2}}+\frac{\omega_{2}^{2} \epsilon_{\omega_{2}} E_{\omega_{2}}}{c^{2}}=-2 \frac{\omega_{2}^{2}}{c^{2}}\left(d^{(2)} E_{\omega_{2}}^{*} E_{2 \omega_{2}}+d^{(2)} E_{\omega_{1}}^{*} E_{\omega_{1}+\omega_{2}}\right. \\
& \left.+d^{(2)} E_{\omega_{1}} E_{\omega_{1}-\omega_{2}}^{*}\right) \\
& \frac{\mathrm{d}^{2}}{\mathrm{~d} z^{2}} E_{2 \omega_{1}}+\frac{4 \omega_{1}^{2} \epsilon_{2 \omega_{1}} E_{2 \omega_{1}}}{c^{2}}=-4 \frac{\omega_{1}^{2}}{c^{2}}\left(d^{(2)} E_{\omega_{1}}^{2}\right. \\
& \left.+d^{(2)} E_{\omega_{1}+\omega_{2}} E_{\omega_{1}-\omega_{2}}\right) \\
& \frac{\mathrm{d}^{2}}{\mathrm{~d} z^{2}} E_{2 \omega_{2}}+\frac{4 \omega_{2}^{2} \epsilon_{2 \omega_{2}} E_{2 \omega_{2}}}{c^{2}}=-4 \frac{\omega_{2}^{2}}{c^{2}}\left(d^{(2)} E_{\omega_{2}}^{2}\right. \\
& \left.+d^{(2)} E_{\omega_{1}+\omega_{2}} E_{\omega_{1}-\omega_{2}}^{*}\right) \\
& \frac{\mathrm{d}^{2}}{\mathrm{~d} z^{2}} E_{\omega_{1}+\omega_{2}}+\frac{\left(\omega_{1}+\omega_{2}\right)^{2} \epsilon_{\omega_{1}+\omega_{2}} E_{\omega_{1}+\omega_{2}}}{c^{2}} \\
& =-2 \frac{\left(\omega_{1}+\omega_{2}\right)^{2}}{c^{2}}\left(d^{(2)} E_{\omega_{1}} E_{\omega_{2}}+d^{(2)} E_{\omega_{1}-\omega_{2}} E_{2 \omega_{2}}\right. \\
& \left.+d^{(2)} E_{\omega_{1}-\omega_{2}}^{*} E_{2 \omega_{1}}\right) \\
& \frac{\mathrm{d}^{2}}{\mathrm{~d} z^{2}} E_{\omega_{1}-\omega_{2}}+\frac{\left(\omega_{1}+\omega_{2}\right)^{2} \epsilon_{\omega_{1}-\omega_{2}} E_{\omega_{1}-\omega_{2}}}{c^{2}} \\
& =-2 \frac{\left(\omega_{1}-\omega_{2}\right)^{2}}{c^{2}}\left(d^{(2)} E_{\omega_{1}} E_{\omega_{2}}^{*}+d^{(2)} E_{\omega_{1}+\omega_{2}} E_{2 \omega_{2}}\right. \\
& \left.+d^{(2)} E_{\omega_{1}+\omega_{2}}^{*} E_{2 \omega_{1}}\right) \text {. }
\end{aligned}
$$

In the system of Eqs. (1), $d^{(2)}$ is the nonlinear optical coefficient. It is assumed to be $100 \mathrm{pm} / \mathrm{V}$ for all processes, a practical but sensible value for many semiconductor materials.

In the undepleted pump regime, all the recombination processes can be neglected, and the formal solution of system (1) is

$$
\begin{gathered}
E_{\omega_{1}}=A_{1} \Phi_{\omega_{1}}^{+}+B_{1} \Phi_{\omega_{1}}^{-}, \\
E_{\omega_{2}}=A_{2} \Phi_{\omega_{2}}^{+}+B_{2} \Phi_{\omega_{2}}^{-}, \\
E_{2 \omega_{1}}=-4 \frac{\omega_{1}^{2}}{c^{2}} \int G_{2 \omega_{1}}(\xi, z) d^{(2)}(\xi) E_{\omega_{1}}^{2}(\xi) \mathrm{d} \xi
\end{gathered}
$$

$$
\begin{gathered}
E_{2 \omega_{2}}=-4 \frac{\omega_{2}^{2}}{c^{2}} \int G_{2 \omega_{2}}(\xi, z) d^{(2)}(\xi) E_{\omega_{2}}^{2}(\xi) \mathrm{d} \xi \\
E_{\omega_{1}+\omega_{2}}=-2 \frac{\left(\omega_{1}+\omega_{2}\right)^{2}}{c^{2}} \int G_{\omega_{1}+\omega_{2}}(\xi, z) d^{(2)}(\xi) E_{\omega_{1}}(\xi) E_{\omega_{2}}(\xi) \mathrm{d} \xi \\
E_{\omega_{1}+\omega_{2}}=-2 \frac{\left(\omega_{1}-\omega_{2}\right)^{2}}{c^{2}} \int G_{\omega_{1}-\omega_{2}}(\xi, z) d^{(2)}(\xi) E_{\omega_{1}}(\xi) E_{\omega_{2}}^{*}(\xi) \mathrm{d} \xi
\end{gathered}
$$

where $\Phi_{\omega}{ }^{+}$and $\Phi_{\omega}{ }^{-}$are the right-to-left (RTL) and left-toright (LTR) linear propagating modes of the structure, ${ }^{21,22}$ respectively, at frequency $\omega$, with $\omega$ $=2 \omega_{1}, 2 \omega_{2}, \omega_{1}+\omega_{2}, \omega_{1}-\omega_{2}$. The propagation modes are steady-state waves, and in each layer they have the form of a superposition of forward- and backward-propagating plane waves (the general solution of the Helmoltz equation), with suitable coefficients that can be calculated through a standard matrix transfer method. ${ }^{23}$ Moreover, the LTR mode satisfies the boundary conditions

$$
\begin{aligned}
1+r^{\mathrm{LTR}} & =\phi^{\mathrm{LTR}}(0), \quad i\left(\frac{\omega}{c}\right)\left(1-r^{\mathrm{LTR}}\right)=\left.\frac{d}{d z} \phi^{\mathrm{LTR}}(z)\right|_{z=0}, \\
t^{\mathrm{LTR}} & =\phi^{\mathrm{LTR}}(L), \quad i\left(\frac{\omega}{c}\right) t^{\mathrm{LTR}}=\left.\frac{d}{d z} \phi^{\mathrm{LTR}}(z)\right|_{z=L},
\end{aligned}
$$

compatible with a plane wave that propagates from left to right. The RTL mode satisfies the boundary conditions

$$
\begin{gathered}
t^{\mathrm{RTL}}=\phi^{\mathrm{RTL}}(0), \quad-i\left(\frac{\omega}{c}\right) t^{\mathrm{RTL}}=\left.\frac{d}{d z} \phi^{\mathrm{RTL}}(z)\right|_{z=0}, \\
1+r^{\mathrm{RTL}}=\phi^{\mathrm{RTL}}(L), \quad i\left(\frac{\omega}{c}\right)\left(-1+r^{\mathrm{RTL}}\right)=\left.\frac{d}{d z} \phi^{\mathrm{RTL}}(z)\right|_{z=L}, \\
i\left(\frac{\omega}{c}\right) t^{\mathrm{LTR}}=\left.\frac{d}{d z} \phi^{\mathrm{LTR}}(z)\right|_{z=L},
\end{gathered}
$$

compatible with a plane wave that propagates from right to left. In Eq. (2), $A_{1}, A_{2}, B_{1}$, and $B_{2}$ are complex coefficients that have the dimensions of an electric field. These coefficients are uniquely determined by the boundary conditions. In the special case of LTR incidence, $B_{1}$ and $B_{2}$ are zero, while $A_{1}$ and $A_{2}$ are the magnitude of the pumps' electric fields at the first interface. On the other hand, in the case of RTL incidence, $A_{1}$ and $A_{2}$ are zero, while $B_{1}$ and $B_{2}$ are the magnitudes of the pumps' electric fields at the last interface. In this section we will study only the case of LTR incidence, so $B_{1}$ and $B_{2}$ are set to zero. In Eq. (2), $G_{\omega}$ is the Green function at frequency $\omega$ $=2 \omega_{1}, 2 \omega_{2}, \omega_{1}+\omega_{2}, \omega_{1}-\omega_{2}:{ }^{21,22}$

$$
G_{\omega}(z, \xi)=\frac{1}{2 i k_{0} t(\omega)}\left\{\begin{array}{ll}
\Phi_{\omega}^{-}(z) \Phi_{\omega}^{+}(\xi) & 0 \leqslant z<\xi \\
\Phi_{\omega}^{+}(z) \Phi_{\omega}^{-}(\xi) & \xi<z \leqslant L
\end{array},\right.
$$

where $t(\omega)$ is the transmission of the structure at frequency $\omega$. 
In the plane-wave regime, the conversion efficiency $\eta$ of a process is defined by the ratio of the intensity of the generated wave $I_{g}$ and the sum of the input pump intensities $I_{\text {pump }}: \eta=I_{g} / \Sigma I_{\text {pump. }}$. In this case, forward $\eta^{+}$and backward $\eta^{-}$conversion efficiencies can be defined without ambiguity as follows: $\eta^{ \pm}=I_{g}^{ \pm} / \Sigma I_{\text {pump }}$, where $I_{g}^{ \pm}$are the intensities of the generated electromagnetic field in the forward (+) and backward (-) directions. Taking into account the relation between the intensity and the electric field for the plane waves (in complex notation) $I=\epsilon_{0} c|E|^{2}$, we have for the conversion efficiencies of the four generated waves

$$
\begin{aligned}
& \eta_{2 \omega_{1}}^{ \pm}=\frac{\pi^{2}}{\epsilon_{0} c} d^{(2)^{2}}\left(\frac{Q}{1+Q}\right)^{2} I_{\text {tot }}\left(\frac{L}{\lambda_{2 \omega_{1}}}\right)^{2} \mid \frac{1}{L} \int_{0}^{L} \operatorname{grating}(z) \\
& \times\left.\left[\Phi_{\omega_{1}}^{+}(z)\right]^{2} \Phi_{2 \omega_{1}}^{\mp}(z) d z\right|^{2}, \\
& \eta_{2 \omega_{2}}^{ \pm}=\frac{\pi^{2}}{\epsilon_{0} c} d^{(2)^{2}}\left(\frac{1}{1+Q}\right)^{2} I_{\text {tot }}\left(\frac{L}{\lambda_{2 \omega_{2}}}\right)^{2} \mid \frac{1}{L} \int_{0}^{L} \operatorname{grating}(z) \\
& \times\left.\left[\Phi_{\omega_{2}}^{+}(z)\right]^{2} \Phi_{2 \omega_{2}}^{\mp}(z) d z\right|^{2} \\
& \eta_{\omega_{1}+\omega_{2}}^{ \pm}=\frac{4 \pi^{2}}{\epsilon_{0} c} d^{(2)^{2}} \frac{Q}{(1+Q)^{2}} I_{\text {tot }}\left(\frac{L}{\lambda_{\omega_{1}+\omega_{2}}}\right)^{2} \mid \frac{1}{L} \int_{0}^{L} \operatorname{grating}(z) \\
& \times\left.\Phi_{\omega_{1}}^{+}(z) \Phi_{\omega_{2}}^{+}(z) \Phi_{\omega_{1}+\omega_{2}}^{\mp}(z) d z\right|^{2}, \\
& \eta_{\omega_{1}-\omega_{2}}^{ \pm}=\frac{4 \pi^{2}}{\epsilon_{0} c} d^{(2)^{2}} \frac{Q}{(1+Q)^{2}} I_{\text {tot }}\left(\frac{L}{\lambda_{\omega_{1}-\omega_{2}}}\right)^{2} \mid \frac{1}{L} \int_{0}^{L} \operatorname{grating}(z) \\
& \times\left.\Phi_{\omega_{1}}^{+}(z)\left[\Phi_{\omega_{2}}^{+}(z)\right]^{*} \Phi_{\omega_{1}-\omega_{2}}^{\mp}(z) d z\right|^{2},
\end{aligned}
$$

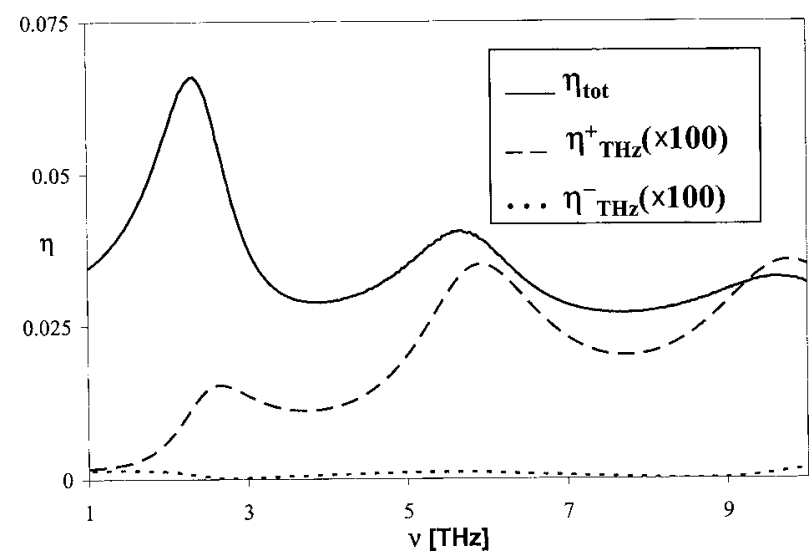

Fig. 2. Conversion efficiency versus generated $\mathrm{THz}$ frequency. The solid curve is the total conversion efficiency, defined as $\eta_{\text {tot }}$ $=\eta_{2 \omega_{1}}+\eta_{2 \omega_{2}}+\eta_{\omega_{1}+\omega_{2}}+\eta_{\omega_{1}-\omega_{2}}$. The long-dashed curve is the forward $\mathrm{THz}$ conversion efficiency $\eta^{+} \mathrm{THz}$ multiplied by 100 . The shortdashed curve is the backward $\mathrm{THz}$ conversion efficiency $\eta_{\mathrm{THz}}^{-}$ multiplied by 100 . The two pumps are arranged as shown in Fig. 3 . The intensity is assumed to be $10 \mathrm{GW} / \mathrm{cm}^{2}$ for each pump.

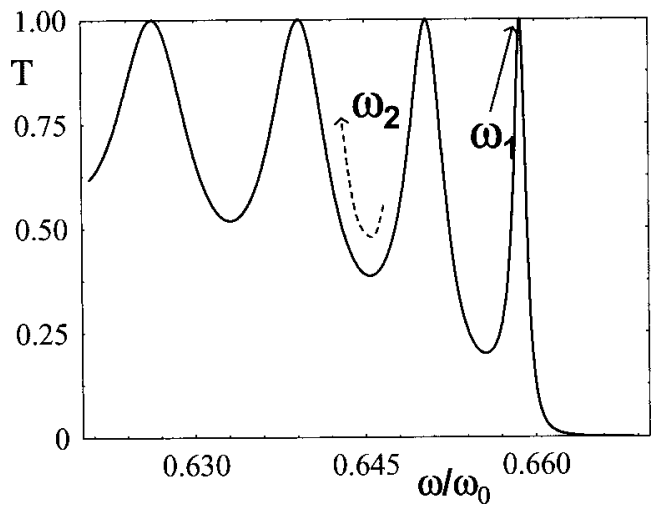

Fig. 3. Transmission spectrum as a function of the normalized frequency near the band edge. $\omega_{1}$ is fixed on the band-edge resonance, whereas $\omega_{2}$ is moved back in frequency.

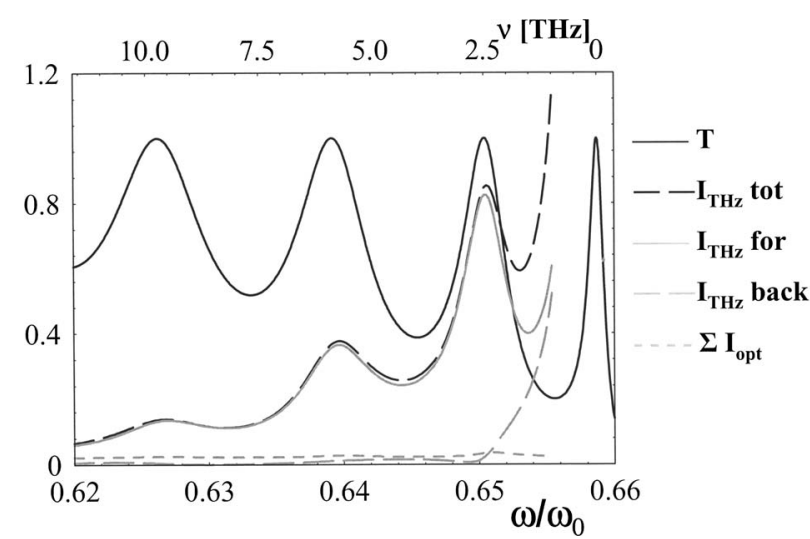

Fig. 4. Transmission and square modulus of the overlap integrals as a function of the second pump's normalized frequency (lower scale) versus the THz-generated frequency (upper scale). Dark curve, the transmission (T); dark dashed curve, the sum of all the $\mathrm{THz}$ overlap integrals $\left(\mathrm{I}_{\mathrm{THz}}\right.$ tot); gray curve, the $\mathrm{THz}$ forward overlap integral ( $\mathrm{I}_{\mathrm{THz}}$ for); gray dashed curve, the backward overlap integral $\left(\mathrm{I}_{\mathrm{THz}}\right.$ back); and gray short-dashed curve, the sum of the overlap integrals of all the other generated frequencies, the optical ones $\left(\Sigma \mathrm{I}_{\mathrm{opt}}\right)$.

where grating $(z)$ is the grating of the second-order nonlinearity. $I_{1}$ is the intensity of the pump at frequency $\omega_{1}, I_{2}$ is the intensity of the second pump, $Q$ is the ratio $I_{1} / I_{2}$, and $I_{\text {tot }}$ is the total intensity $I_{1}+I_{2}$. From Eq. (4), it follows that we have the higher conversion efficiency for the difference frequency (and the sum frequency) when the two pumps are balanced $\left(I_{1}=I_{2}\right)$. So we will consider only the case $I_{1}=I_{2}$. The total conversion efficiency $\eta_{\text {tot }}$ is defined as the sum of all the conversions efficiencies: $\eta_{\text {tot }}=\Sigma \eta_{i}$. In Fig. 2 we report $\eta_{\text {tot }}$ as a function of the generated $\mathrm{THz}$ radiation. The total intensity is fixed to a value of $20 \mathrm{GW} / \mathrm{cm}^{2}\left(10 \mathrm{GW} / \mathrm{cm}^{2}\right.$ for each pump). $\omega_{1}$ is tuned at the band edge, whereas $\omega_{2}$ is chosen such that $\left(\omega_{1}-\omega_{2}\right)$ ranges from 1 to approximately $10 \mathrm{THz}$ (see Fig. 3 ) and in such a way that the second harmonic always falls inside the second-order gap to suppress its conversion efficiency. As shown in Fig. 2, the undepleted pump approximation is well verified also for the relatively intense, incident fields we are considering. The conversion efficiencies of Eqs. (4) are proportional to the product of the square of the frequency $(L / \lambda)^{2}$ and a term that represents the structure's properties via an overlap integral that we focus on below: 
$I^{ \pm}=\left|\frac{1}{L} \int_{0}^{L} \operatorname{grating}(z) \Phi_{\omega_{1}}^{+}(z)\left[\Phi_{\omega_{2}}^{+}(z)\right]^{*} \Phi_{\omega_{1}-\omega_{2}}^{\mp}(z) d z\right|^{2}$.

In Fig. 4 we report the behavior of the integral overlap as a function of $\omega_{2}$ (lower scale) and the generated $\mathrm{THz}$ fre-
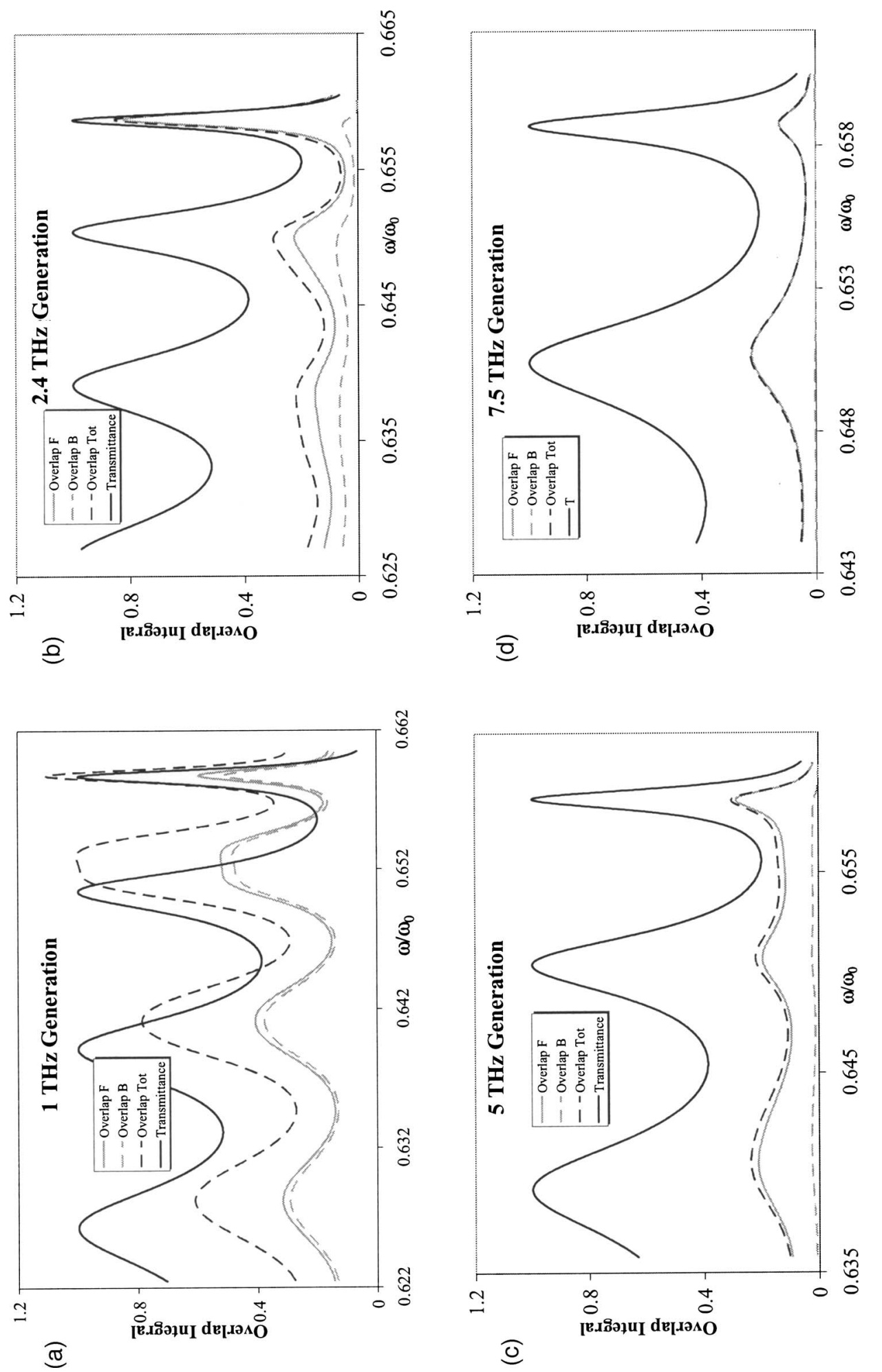

Fig. 5. Overlap integrals as functions of the normalized first pump frequency $\omega_{1} / \omega_{0}$, for four generated frequencies: (a) $\omega_{1}-\omega_{2}$ $=1 \mathrm{THz}$, (b) $\omega_{1}-\omega_{2}=2.4 \mathrm{THz}$, (c) $\omega_{1}-\omega_{2}=5 \mathrm{THz}$, (d) $\omega_{1}-\omega_{2}=7.5 \mathrm{THz}$. Black curve, the transmission; black dashed curve, the sum of the THz overlap integrals (Overlap Tot); gray curve, the forward THz overlap integral (Overlap F); gray dashed curve, the backward overlap integral (Overlap B). 
low $\mathrm{THz}$ frequency $(\sim 1 \mathrm{THz})$, better results can be obtained with a structure having broader resonances, such that both pumps can be tuned within the same resonance. For example, with a similar structure (25 periods instead of 40) the overlap integrals at $1 \mathrm{THz}$ (and so the conversion efficiency) are nearly double. Moreover, the structure (at least for LTR incidence) will generate efficiently up to 3-4 THz, as the overlap integral becomes progressively smaller at higher frequencies.

Up to now we have used a fixed value of $\omega_{1}$ to maximize its localization properties while tuning $\omega_{2}$ to lower frequencies. This may not be the best choice, but it provides a good qualitative picture of what one may expect for structures similar to our own. If the desired outcome is a tunable device with only one tunable pump, the situation we have described is suitable. In Fig. 5 we show what happens to the overlap integrals if we tune $\omega_{1}$ away from the band edge, for the generation of $1,2.4,5$, and $7.5 \mathrm{THz}$. The result is that only for the highest frequency $(7.5 \mathrm{THz})$ the chosen configuration is not ideal. The plane-wave approach offers a complete description for all the optical frequencies because it allows the calculation of the conversion efficiency as long as the spatial properties of the emitted fields are those of plane waves. In fact, this model offers only partial information on $\mathrm{THz}$ emission in that, although it allows a correct estimation of the conversion efficiency, diffraction of the generated $\mathrm{THz}$ waves is not taken into account, and we have little or no information on the spatial distribution of the radiation. This aspect of $\mathrm{THz}$ generation will be discussed in Section 4 with the help of a 3-D model.

\section{THREE-DIMENSIONAL APPROACH}

In this section we develop a 3-D model to simulate the spatial characteristic of $\mathrm{THz}$ generation. The $\mathrm{THz}$ signal is generated from the interaction of two optical pumps in a volume that can be approximated with a cylinder of radius $R$ (equal to the pumps' spot size) and length equal to the length of the 1-D PC structure (See Fig. 6). As explained in Section 3, we can neglect all the recombination terms. The THz field, according to Maxwell's equations, satisfies the vectorial equation

$$
\nabla \times \nabla \times \mathbf{E}-\omega^{2} \mu_{0} \hat{\epsilon} \mathbf{E}=2 \epsilon_{0} \overline{\bar{d}}^{(2)}: \mathbf{E}_{\mathbf{1}} \mathbf{E}_{2}^{*}
$$

Equation (6) has the formal solution

$$
\mathbf{E}=\mu_{0} \omega^{2} \int_{V} \mathrm{~d} V^{\prime} \hat{G}\left(\mathbf{r}, \mathbf{r}^{\prime}\right) 2 \epsilon_{0} \overline{\bar{d}}^{(2)}: \mathbf{E}_{\mathbf{1}} \mathbf{E}_{2}^{*}
$$

where $\hat{G}\left(\mathbf{r}, \mathbf{r}^{\prime}\right)$ is the dyadic Green function of the problem; $\overline{\bar{d}}^{(2)}$ is the nonlinear tensor; $\mathbf{E}_{\mathbf{1}}$ and $\mathbf{E}_{\mathbf{2}}$ are the pump fields. Without lack of generality we will study the configuration $\mathbf{E}_{1} / / \mathbf{E}_{1} / / \mathbf{P}^{N L}$. As the $\mathrm{THz}$ wavelength is much greater compared with the length of the structure, the structure itself may be considered a point source of $\mathrm{THz}$ radiation. In Eq. (7) we can use the free-space dyadic Green function. ${ }^{24}$ In the far-field approximation, the dyadic Green function reduces to the simple form ${ }^{25}$

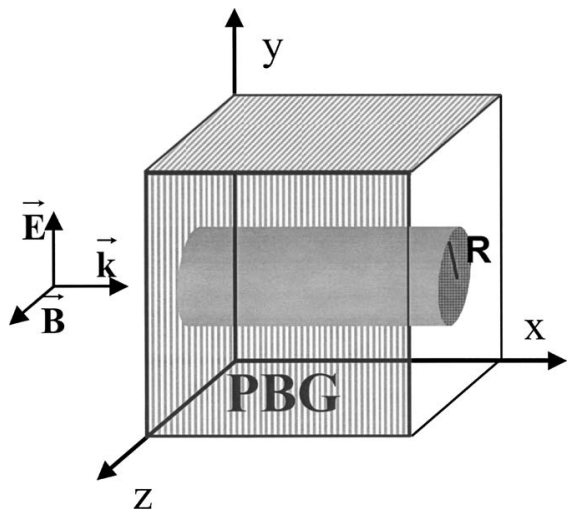

Fig. 6. Configuration of the 3-D simulations. The pumps enter the structure at normal incidence; the interactive area is a cylinder of radius $R$ equal to the spot size and length $L$ equal to the photonic crystal length.-PBG,

$$
G_{i j}\left(\mathbf{r}, \mathbf{r}^{\prime}\right)=\left(\delta_{i j}-x_{i} x_{j}\right) \frac{1}{4 \pi r^{3}} \exp \left(i k_{0} r\right) \exp \left(-i k_{0} \frac{\mathbf{r} \cdot \mathbf{r}^{\prime}}{r}\right) .
$$

Assuming a plane-wave form for the pump fields from Eqs. (6) and (7), we arrive at the following expression for the electric field:

$$
\begin{aligned}
\mathbf{E}= & \mu_{0} \epsilon_{0} \omega^{2} d^{(2)} A_{1} A_{2}^{*} \exp \left(i k_{0} r\right) \\
& \times \exp (i \omega t) \frac{R}{k_{0} \rho} J_{1}\left(\frac{k_{0} \rho R}{r}\right) I_{x}\left(\begin{array}{c}
-\frac{x y}{r^{2}} \\
1-\frac{y^{2}}{r^{2}} \\
-\frac{y z}{r^{2}}
\end{array}\right),
\end{aligned}
$$

where $I_{x}=\int_{0}^{L} \mathrm{~d} x^{\prime} \exp \left\{-i k_{0}\left[\left(x x^{\prime}\right) / r\right]\right\} \Phi_{1}^{+}\left(x^{\prime}\right) \Phi_{2}^{+}\left(x^{\prime}\right)^{*}$ grating $\left(x^{\prime}\right), J_{1}$ is the Bessel function of order $1, \rho^{2}=y^{2}+z^{2}$, and $A_{1}$ and $A_{2}$ are the amplitudes of the pump fields $\mathbf{E}_{\mathbf{1}}$ and $\mathbf{E}_{\mathbf{2}}$.

From Eq. (9) the square modulus of the electric field and the Poynting vector $(\mathbf{S})$ can easily be calculated. The intensity of the electromagnetic field is given by the modulus of the Poynting vector. It comes out that the emission is directional, namely, the intensity of the electromagnetic field is largest near the $x$ axis and the direction of the Poynting vector is nearly along the $x$ axis. Moreover, even if the emission is not in the form of a plane wave, we have that $|\mathbf{S}| \propto \mathbf{E} \cdot \mathbf{E}^{\prime}$.

The $\mathrm{THz}$ emission obtained by pumping the 1-D PC structure may be compared with the emission obtained from an equivalent bulk and that of an equivalent dipole. In Fig. 7 we show both bulk and equivalent dipole emissions for the generation of $1,2.5,5$, and $7.5 \mathrm{THz}$.

The equivalent bulk is composed of a nonlinear material of length $L 1$ equal to the sum of all the nonlinear layers in the 1-D PC structure. The emission from that structure is found following the model developed by Shen. ${ }^{25}$

The equivalent dipole is a point dipole of intensity given by the sum of all the dipoles distributed in the 1-D PC structure. Although the point dipole is an idealization, comparing this case with the 1-D PC and the bulk shows 
that the 1-D PC and the bulk have a much more directional emission. That directionality is due to the fact that in both cases the dipoles are excited by highly directional optical pumps. As predicted by the plane-wave approach, the emission from the 1-D PC structure can be much higher than the emission from the bulk. In particular, at 1 and $2.5 \mathrm{THz}$, Figs. 7(a) and 7(b), we have an enhancement of nearly 6 with respect to the bulk. At $5 \mathrm{THz}$ the emission from the 1-D PC and from the bulk are nearly equal. At 7.5 THz there is no advantage in using the 1-D PC.

To evaluate the conversion efficiency, we calculate the flux of the Poynting vector through a close surface and divide it by the flux of the pumps through the input surface $S$ (a circle of radius $R$ ). For example, we can enclose the source in a cylinder of radius $a$ and length $2 d$, where $d$ $\gg L$ (far-field approximation). In that case we have

$$
\eta=\frac{\int_{0}^{a} \mathrm{~d} \rho \rho \int_{0}^{2 \pi} \mathrm{d} \varphi S_{x}(d, \rho, \varphi)-\int_{0}^{a} \mathrm{~d} \rho \rho \int_{0}^{2 \pi} \mathrm{d} \varphi S_{x}(-d, \rho, \varphi)+a \int_{-d}^{d} \mathrm{~d} x^{\prime} \int_{0}^{2 \pi} \mathrm{d} \varphi\left[S_{y}\left(x^{\prime}, a, \varphi\right) \cos \varphi+S_{z}\left(x^{\prime}, a, \varphi\right) \sin \varphi\right]}{\pi R^{2} \epsilon_{0} c\left(A_{1}^{2}+A_{2}^{2}\right)} .
$$

Note that the Poynting vector goes to zero far from the $x$ axis. So if in Eq. (10) the radius a is taken large enough to contain the peak of the emission, we identify three contributions to the conversion efficiency: forward $\left(\eta^{+}\right)$, backward $\left(\eta^{-}\right)$, and lateral $\left(\eta^{\text {lat }}\right)$ conversion efficiencies, as follows:

$$
\begin{gathered}
\eta^{+}=\frac{\int_{0}^{a} \mathrm{~d} \rho \rho \int_{0}^{2 \pi} \mathrm{d} \varphi S_{x}(d, \rho, \varphi)}{\pi R^{2} \epsilon_{0} c\left(A_{1}^{2}+A_{2}^{2}\right)}, \\
\eta^{-}=\frac{-\int_{0}^{a} \mathrm{~d} \rho \rho \int_{0}^{2 \pi} \mathrm{d} \varphi S_{x}(-d, \rho, \varphi)}{\pi R^{2} \epsilon_{0} c\left(A_{1}^{2}, A_{2}^{2}\right)},
\end{gathered}
$$

(a)

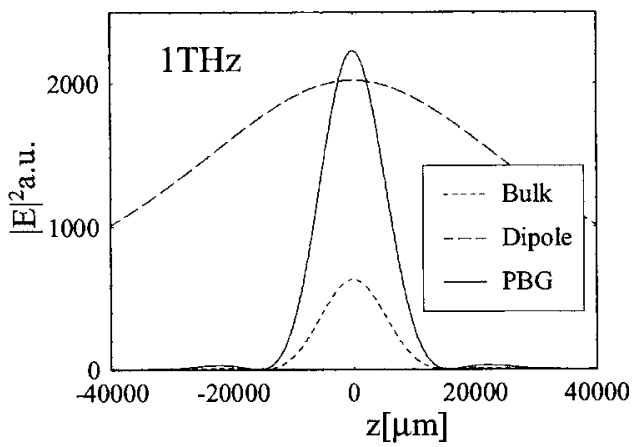

(c)

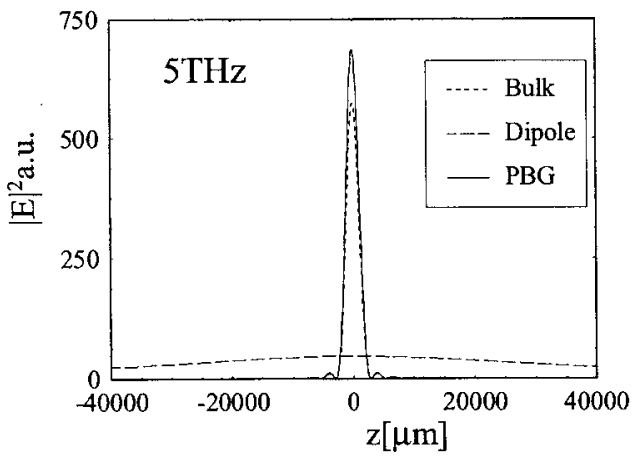

$$
\eta^{\text {lat }}=\frac{a \int_{-d}^{d} \mathrm{~d} x^{\prime} \int_{0}^{2 \pi} \mathrm{d} \varphi\left[S_{y}\left(x^{\prime}, a, \varphi\right) \cos \varphi+S_{z}\left(x^{\prime}, a, \varphi\right) \sin \varphi\right]}{\pi R^{2} \epsilon_{0} c\left(A_{1}^{2}+A_{2}^{2}\right)} .
$$

The forward and the backward conversion efficiencies are proportional to the flux of the Poynting vector through the basis of the cylinder. The lateral conversion efficiency is proportional to the flux of the Poynting vector through the lateral surface of the cylinder.

It turns out that the emission through the lateral surface is negligible with respect to the emission through the basis of the cylinder. Expressions (11) can be recast in the following form:
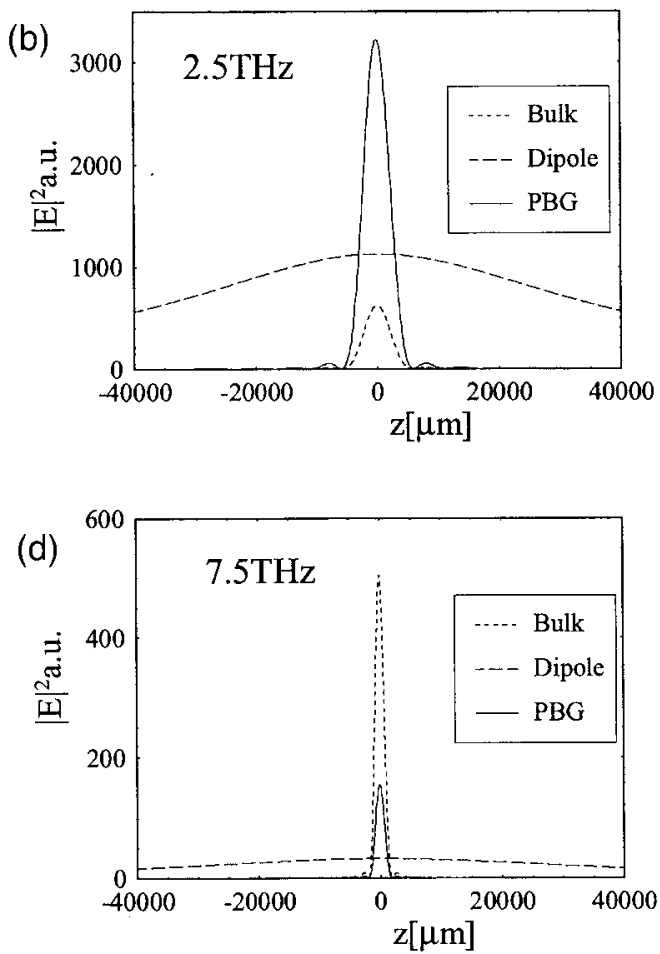

Fig. 7. Square modulus of the electric field, at a plane parallel to the $y z$ plane, with distance of $4 \mathrm{~cm}$ from the source, in the forward direction as a function of the $z$ coordinate. Solid curves, the photonic crystal emission; dashed curves, the equivalent dipole emission; short-dashed curves, the equivalent bulk emission. (a) $\omega_{1}-\omega_{2}=1 \mathrm{THz}$, (b) $\omega_{1}-\omega_{2}=2.5 \mathrm{THz}$, (c) $\omega_{1}-\omega_{2}=5 \mathrm{THz}$, and (d) $\omega_{1}-\omega_{2}=7.5 \mathrm{THz}$. 


$$
\begin{aligned}
& \eta^{+}=\frac{4 \pi^{2}}{\epsilon_{0} c} d^{(2)^{2}} \frac{Q}{(1+Q)^{2}} I_{\text {tot }}\left(\frac{L}{\lambda}\right)^{2} I_{\text {eq }}^{+}, \\
& \eta^{-}=\frac{4 \pi^{2}}{\epsilon_{0} c} d^{(2)^{2}} \frac{Q}{(1+Q)^{2}} I_{\text {tot }}\left(\frac{L}{\lambda}\right)^{2} I_{\text {eq }}^{-}
\end{aligned}
$$

with

$$
\begin{aligned}
I_{\text {eq }}^{+}= & \frac{1}{\pi L^{2}} \int_{s} \frac{1}{y^{2}+z^{2}} \frac{1}{r^{4}}\left(J_{1}\right)^{2}\left[r a\left(a^{2}+z^{2}\right)\left|I_{x}\right|^{2}\right. \\
& \left.+z^{2} r \operatorname{Re}\left(I_{x} L_{x}^{*}\right)\right] \mathrm{d} s, \\
I_{\text {eq }}^{-}= & \frac{-1}{\pi L^{2}} \int_{s} \frac{1}{y^{2}+z^{2}} \frac{1}{r^{4}}\left(J_{1}\right)^{2}\left[-r a\left(a^{2}+z^{2}\right)\left|I_{x}\right|^{2}\right. \\
& \left.+z^{2} r \operatorname{Re}\left(I_{x} L_{x}^{*}\right)\right] \mathrm{d} s .
\end{aligned}
$$

In Eqs. (13) $L_{x}=\int_{0}^{L} \mathrm{~d} x^{\prime} x^{\prime} \exp \left[-i k_{0}\left(x x^{\prime} / r\right)\right] \Phi_{1}^{+}\left(x^{\prime}\right) \Phi_{2}^{+}\left(x^{\prime}\right)^{*}$ grating $\left(x^{\prime}\right)$. The $a$-dimensional quantities $I_{\text {eq }}^{+}$and $I_{\text {eq }}^{-}$play in the conversion efficiency of the 3-D model the same role as the overlap integrals defined in Eq. (5).

In Fig. 8 we compare the behavior of the overlap integrals in the two models. Although the two models have the same qualitative behavior, there are some quantitative differences owing to the fact that, on the one hand, the 3-D model neglects the internal details of the structure for the $\mathrm{THz}$ frequency, whereas, on the other hand, the 1-D model neglects the 3-D aspect of the emission. The localization of the field grows with frequency, while the emission spread goes in the other direction. So, at higher frequencies, the field localization becomes dominant, and the 1-D model makes more accurate predictions, whereas at lower frequencies it is the $3-\mathrm{D}$ model that becomes more accurate. $\mathrm{THz}$ frequencies lie in the transition region between those two regimes. In Fig. 9 we plot the field localization (the square modulus of the 1-D PC modes) moving from the microwaves to the far infra-

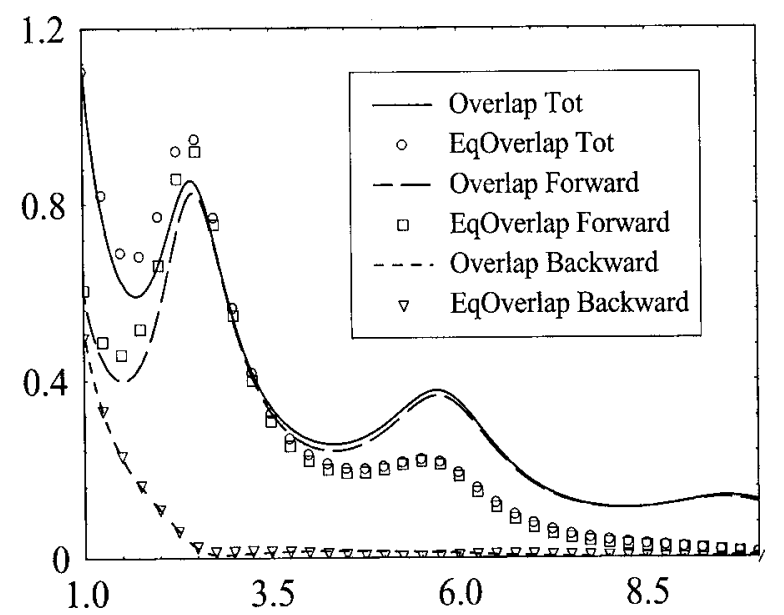

Fig. 8. Overlap integrals (curves) and their equivalents for the 3-D model (points) as functions of the generated $\mathrm{THz}$ frequency. Solid curve and circles, the total (sum of the forward and backward) overlaps (Overlap Tot and EqOverlap Tot); dashed curve and squares, the forward overlaps (Overlap Forward and EqOverlap Forward); short-dashed curve and triangles, the backward overlaps (Overlap Backward and EqOverlap Backward).

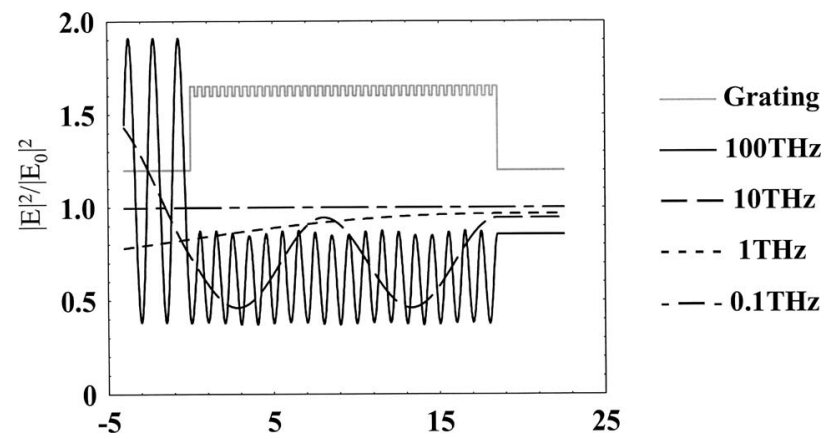

Fig. 9. Field localization as a function of the propagation coordinate $x$, for several incidence frequencies $\nu$, in the plane-wave approximation. Solid curve, $\nu=100 \mathrm{THz}$; dashed curve, $\nu$ $=10 \mathrm{THz}$; short-dashed curve, $\nu=1 \mathrm{THz}$; dashed-dotted curve, $\nu$ $=0.1 \mathrm{THz}$. Gray curve, the refractive index grating of the structure.

red. If there is no localization, we will have a constant value of 1 ; otherwise, we will see oscillations and may be high-localization zones. It is clear that, from this point of view, $\mathrm{THz}$ frequencies represent a transition region. Even if we do not have strong-field localization, one cannot assume that $\mathrm{THz}$ radiation is not affected by the structure.

\section{FOUR-GATE SYSTEM}

As shown by Centini et al., ${ }^{26}$ the use of the 1-D PC structure as a four-gate system, namely, the structure is pumped from both sides, modifies the field distribution of the pumps inside the structure. This effect allows us to change the conversion efficiency ${ }^{26}$ and the ratio of the forward and backward emissions by changing the phase difference between the RTL and LTR input fields. The overlap integrals [Eqs. (5) and (13)] become functions of the phase differences $\Delta \varphi_{1}$ and $\Delta \varphi_{2}$ between the RTL and LTR incident fields at frequencies $\omega_{1}$ and $\omega_{2}$, respectively. In particular, pumping from both sides makes it possible to control (a) field localization in the active layers and (b) the amount of relative forward and backward $\mathrm{THz}$ emissions. To take advantage of effect (a), we need the pumps to be tuned at the transmission peaks near the band edge, where the field is most intense. To take advantage of effect (b), we need the LTR and RTL modes of the generated frequency to have different localization properties.

In Fig. 10 we report field localization of the structure's modes (LTR and RTL) for 1, 2.5, 5, and 7.5 THz. At $1 \mathrm{THz}$ the field is not affected by the structure: There is no field localization, and the LTR and RTL are practically the same and flat. In this case we may not change the balance of the forward and backward emissions. Moreover, the two pumps are tuned at the band edge, and we expect to have higher conversion efficiency. At $2.5 \mathrm{THz}$, LTR and RTL mode localizations are sensibly different. We expect to be able to change the balance of the forward and backward emissions in an equally sensible way. In that only one pump is tuned at the band edge, we do not expect to fully exploit field localization effects. At 5 and $7.5 \mathrm{THz}$ the LTR and RTL modes are still localized differently, and so we can partially control the directionality of the emission. But the efficiency of the process will not be as high as also having the second pump tuned far from the band edge. 
(a)

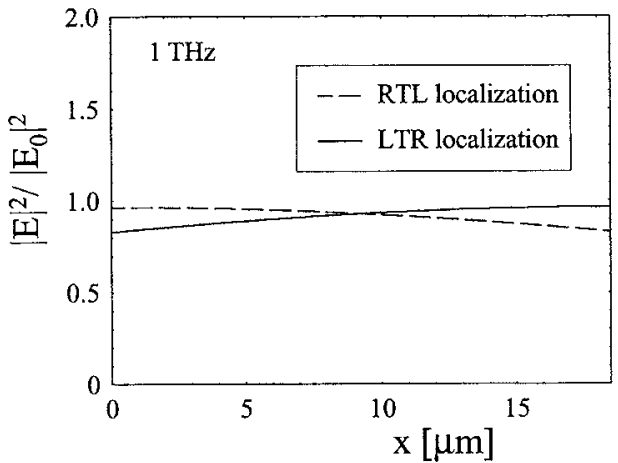

(c)

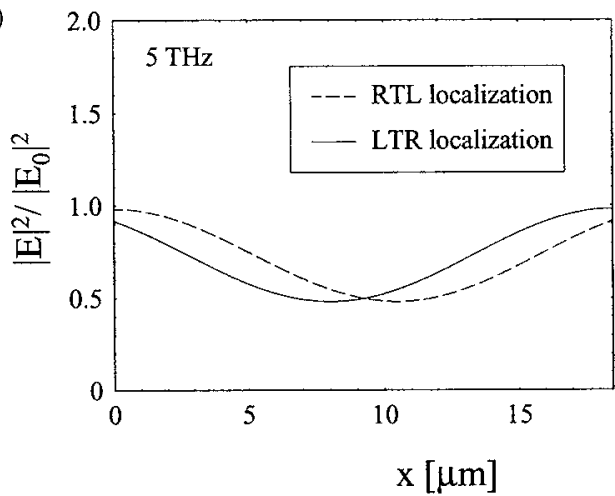

(b)

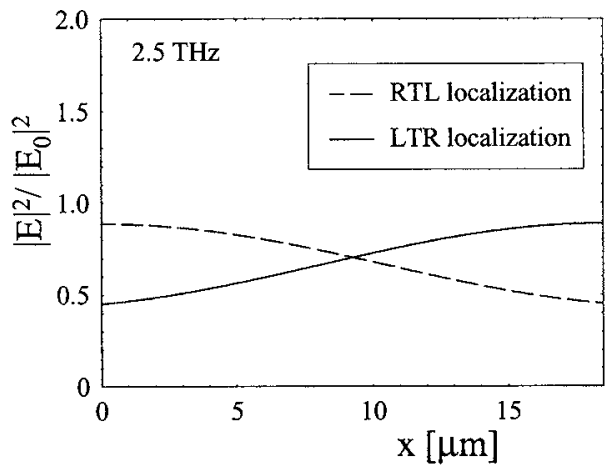

(d)

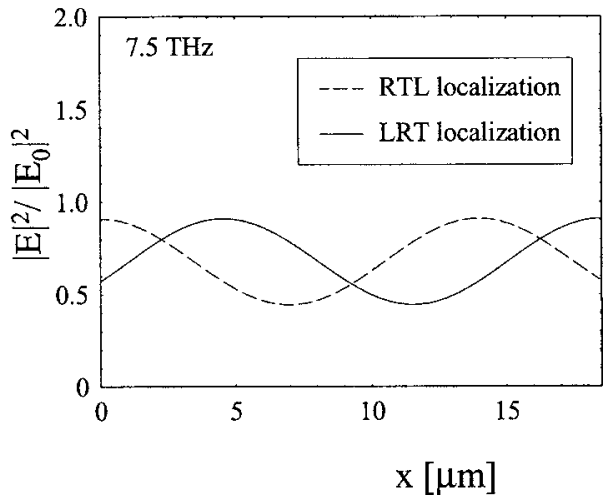

Fig. 10. Field localization as a function of the propagation coordinate $x$, in the case of LTR incidence (solid curves) and RTL incidence (dashed curves). The frequency $\nu$ is (a) $1 \mathrm{THz}$, (b) $2.5 \mathrm{THz}$, (c) $5 \mathrm{THz}$, (d) $7.5 \mathrm{THz}$.

In the conversion efficiency formulas, in the case of LTR plus RTL incidence, the only factors that change are the overlap integrals, Eq. (5), and the corresponding quantity in the 3-D model, Eqs. (13). In Eqs. (5) and (13), $\Phi_{\omega_{1}}^{+}(z)$ must be replaced by $\Phi_{\omega_{1}}^{+}(z)+\Phi_{\omega_{1}}^{-}(z) \exp \left(i \Delta \varphi_{1}\right)$, and $\Phi_{-\omega_{2}}^{+}(z)$ must be changed to $\Phi_{-\omega_{2}}^{+\omega_{1}}(z)+\Phi_{-\omega_{2}}^{-1}(z) \exp \left(i \Delta \varphi_{2}\right)$. Because in the case of symmetric or nearly symmetric structures, $\phi_{\omega}^{+}(z) \approx \phi_{\omega}^{-}(L-z)$, the following condition is satisfied: $I^{-}\left(-\Delta \varphi_{1},-\Delta \varphi_{2}\right)=I^{+}\left(\Delta \varphi_{1}, \Delta \varphi_{2}\right)$

This means that if the behavior of the forward overlap integral is known, then the backward overlap integral is also known. The phase differences $\Delta \varphi_{1}$ and $\Delta \varphi_{2}$ can be chosen (at each frequency) in order to maximize the total conversion efficiency $\left(\eta^{+}+\eta^{-}\right)$, namely, the sum of the forward and backward overlap integrals, as well as the forward or the backward conversion efficiency.

In Fig. 11 we show what happens when $\Delta \varphi_{1}$ and $\Delta \varphi_{2}$ are chosen in order to maximize the total conversion efficiency. Note that $\Delta \varphi_{1}$ and $\Delta \varphi_{2}$ have a different value for each frequency. The forward and the backward overlap integrals, as well as the sum of the two, are plotted as functions of the generated $\mathrm{THz}$ frequency. Comparing the behavior of the total overlap integral with the case of LTR incidence (Fig. 8), we find an enhancement factor of nearly 4 at $1 \mathrm{THz}$, which translates to an enhancement factor of nearly 20 with respect to the bulk. Moreover, the four-gate system is always more efficient than the onesided incidence, but any significant advantage quickly goes away as the second pump moves away from the band edge, and the generated frequency increases. The maximum efficiency is reached almost always when the forward and the backward emissions are nearly equal. The

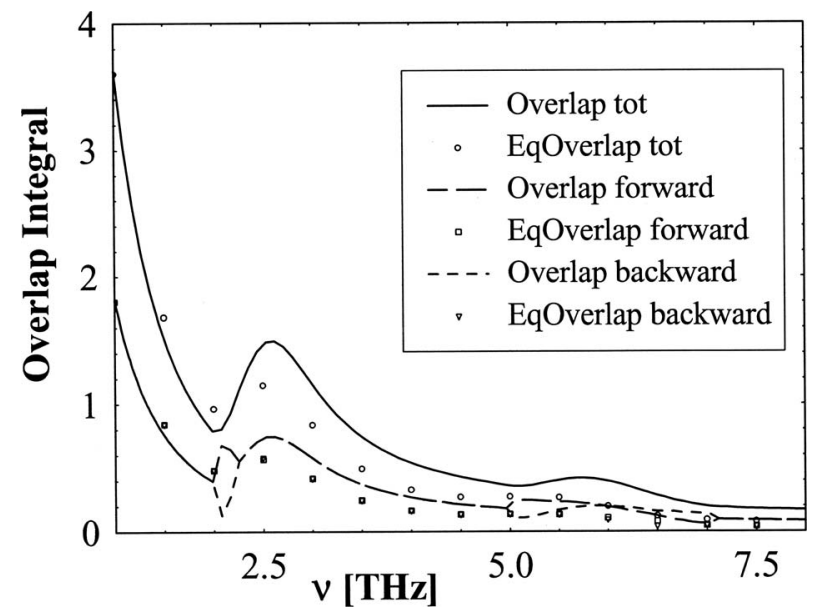

Fig. 11. Overlap integrals (curves) and their equivalents for the 3-D model (points) in the case of both sides' incidence. For both pumps, the phase difference between the LTR and the RTL beams is chosen in order to maximize the total conversion efficiency. Solid curve and circles, the total overlaps; the dashed curve and squares, the forward overlaps; the short-dashed curve and the triangles, the backward overlaps.

3-D and the 1-D models show some quantitative differences for reasons that were discussed earlier. In Fig. 12 we show what happens when $\Delta \varphi_{1}$ and $\Delta \varphi_{2}$ are chosen in order to maximize the forward conversion efficiency. According to Fig. 10, near $1 \mathrm{THz}$, we cannot control the direction of the emission. In that case forward and backward emissions are similar. As one moves to higher frequencies, control of the directionality of the emission increases, and the efficiency drops. 


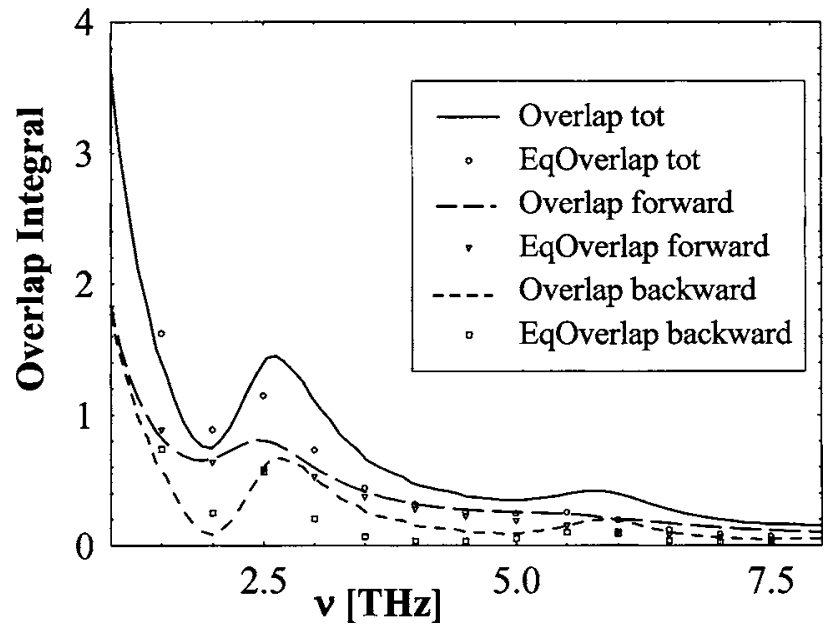

Fig. 12. Overlap integrals (curves) and their equivalents for the 3 -D model (points) in the case of both sides' incidence. For both pumps, the phase difference between the LTR and the RTL beams is chosen in order to maximize the forward conversion efficiency. Solid curve and circles, the total overlaps; the dashed curve and squares, the forward overlaps; the short-dashed curve and the triangles, the backward overlaps.

\section{CONCLUSION}

We have developed a 3-D model able to simulate $\mathrm{THz}$ emission from a photonic crystal structure via differencefrequency generation. The model allows the calculation of the conversion efficiency as well as the spatial properties of the emission. This model also shows that 1-D PC structures are versatile and powerful devices, able to efficiently generate $\mathrm{THz}$ frequencies. The general analysis on $\mathrm{THz}$ emission in a photonic crystal structure carried out in this study is a useful overview that will allow us to properly design structures for specific purposes.

In this paper we have not referred to any particular material, and so our results have general validity, thanks in part to the fact that 1-D PCs with similar characteristics can be obtained by one's adjusting the number of periods or the index contrast or both. A structure with low index contrast and large number of periods behaves like a structure with a high index contrast and fewer periods. Moreover, we stress that not all materials suitable for stratification have been characterized in the $\mathrm{THz}$ regime, and, at the present time, the best candidate for nonlinear material appears to be GaAs, which has already been used to generate $\mathrm{THz}$ pulses via optical rectification. ${ }^{5}$

Corresponding author M. Scalora can be reached by e-mail at michael.scalora@us.army.mil.

\section{REFERENCES}

1. N. M. Froberg, B. B. Hu, X.-C. Zhang, and D. H. Auston, "Terahertz radiation from a photoconducting antenna array," IEEE J. Quantum Electron. 28, 2291-2301 (1992).

2. Y. C. Shen, P. C. Upadhya, E. H. Linfield, and H. E. Beere, "Terahertz generation from coherent optical phonons in biased GaAs photoconductive emitter," Phys. Rev. E 69, 235325 (2004).

3. L. Xu, X.-C. Zhang, and D. H. Auston, "Terahertz beam generation by femtosecond optical pulses in electrooptic materials," Appl. Phys. Lett. 61, 1784-1787 (1992).

4. K. H. Yang, P. L. Richards, and Y. R. Shen, "Generation of far-infrared radiation by picosecond light pulses in $\mathrm{LiNbO}_{3}$," Appl. Phys. Lett. 19, 320-323 (1971).

5. A. S. Nikoghosyan, E. M. Laziev, R. M. Martirosyan, and A. A. Hakhoumian, "Efficient ultrashort light pulse conversion in $\mathrm{GHz}-\mathrm{THz}$ pulses in $\mathrm{ZnTe}, \mathrm{GaAs}$, and DAST crystals," iCONO 2001: Ultrafast Phenomena and Strong Laser Fields, V. M. Gordienko, A. A. Afanas'ev, and V. V. Shuvalov, eds., Proc. SPIE 4752, 40-48 (2002).

6. A. Nahata, D. H. Auston, C. Wu, and J. T. Yardley, "Generation of terahertz radiation from poled polymer," Appl. Phys. Lett. 67, 1358-1361 (1995).

7. A. Tredicucci, F. Capasso, G. Gmachi, D. L. Sivco, A. L. Hutchinson, and A. Y. Chao, "High performance interminiband quantum cascade lasers with grades superlattices," Appl. Phys. Lett. 73, 2101-2103 (1998).

8. M. A. Piestrup and R. N. Fleming, "Continuosusly tunable submillimeter wave source,” Appl. Phys. Lett. 26, 418-421 (1975).

9. A. M. Weiner and D. E. Leaird, "Generation of terahertzrate trains of femtosecond pulses by phase-only filtering," Opt. Lett. 15, 51-53 (1990).

10. T. Taniuchi and H. Nakanishi, "Continuously tunable terahertz-wave generation in $\mathrm{GaP}$ crystal by collinear difference frequency mixing," Electron. Lett. 40, 327-328 (2004).

11. W. Shi and Y. J. Ding, "A monochromatic and high-power terahertz source tunable in the ranges of 2.7-38.4 and $58.2-3540 \mu \mathrm{m}$ for variety of potential application," Appl. Phys. Lett. 84, 1635-1638 (2004).

12. T. Taniuchi, H. Adachi, S. Okda, T. Sasaki, and H. Nakanishi, "Continuously tunable $\mathrm{THz}$ and far-infrared wave generation fron DAST crystal," Electron. Lett. 40, 549-551 (2004).

13. P. E. Powers, R. A. Alkuwari, J. W. Haus, K. Suizu, and H. Ito, "Terahertz generation with tandem seeded optical parametric generators," Opt. Lett. 30, 640-642 (2005).

14. M. Bertolotti, C. M. Bowden, and C. Sibilia, "Nanoscale linear and nonlinear optics," AIP Conf. Proc. 560, 1-31 (2001).

15. Y. Lu, M. Xiao, and G. J. Salamo, "Coherent microwave generation in nonlinear photonic crystal," IEEE J. Quantum Electron. 38, 481-485 (2002).

16. N. Bloembergen and A. J. Sievers, "Nonlinear optical properties of periodic laminar structures," Appl. Phys. Lett. 17, 483-486 (1970).

17. G. D’Aguanno, M. Centini, M. Scalora, C. Sibilia, M. Bertolotti, M. J. Bloemer, and C. M. Bowden, "Generalized coupled-mode theory for $\chi^{(2)}$ interactions in finite multilayered structures,” J. Opt. Soc. Am. B 19, 2111-2121 (2002).

18. M. Centini, G. D’Aguanno, C. Sibilia, L. Sciscione, M. Bertolotti, M. Scalora, and M. J, Bloemer, "Non-phasematched enhancement of second-harmonic generation in multilayer structures with internal reflections," Opt. Lett. 29, 1924-1926 (2004)

19. J. W. Haus, P. Powers, M. Torres-Cisneros, M. Scalora, M. J. Bloemer, N. Akozbek, and M. A. Meneses-Nava, "Enhancement tunable terahertz generation in photonic band-gap structures," Laser Phys. 14, 635-642 (2004).

20. G. D’Aguanno, M. Centini, M. Scalora, C. Sibilia, M. J. Bloemer, C. M. Bowden, J. W. Haus, and M. Bertolotti, "Group velocity, energy velocity, and superluminal propagation in finite photonic band-gap structures," Phys. Rev. E 63, 036610 (2001).

21. O. Di Stefano, S. Savasta, and R. Girlanda, "Mode expansion and photon operators in dispersive and absorbing dielectrics," J. Mod. Opt. 48, 67-84 (2001).

22. G. D’Aguanno, N. Mattiucci, M. Scalora, M. J. Bloemer, 
and A. M. Zheltikov, "Density of modes and tunneling times in finite, one-dimensional, photonic crystals: a comprehensive analysis," Phys. Rev. E 70, 016612 (2004).

23. J. Lekner, Theory of Reflection (Martin Nijhoff, 1987), Chap. 12.

24. W. C. Chew, Waves and Fields in Inhomogeneous Media (Van Nostrand Reinhold, 1990), pp. 375-378.
25. Y. R. Shen, The Principle of Nonlinear Optics (Wiley, 1984), pp. 110-113.

26. M. Centini, G. D’Aguanno, M. Scalora, M. J. Bloemer, C. M. Bowden, C. Sibilia, N. Mattiucci, and M. Bertolotti, "Dynamics of counterpropagating pulses in photonic crystals: enhancement and suppression of stimulated emission processes," Phys. Rev. E 67, 036617 (2003). 